\title{
What should we focus on before preimplantation genetic diagnosis/screening?
}

\author{
Zhong Zheng ${ }^{1,2}$, Xiaoming Zhao ${ }^{1,2}$, Bing $\mathrm{Xu}^{1,2}$, Ning Yao ${ }^{1,2}$
}

\author{
${ }^{1}$ Center for Reproductive Medicine, Ren Ji Hospital, School of Medicine, Shanghai Jiao \\ Tong University, Shanghai, China \\ ${ }^{2}$ Shanghai Key Laboratory for Assisted Reproduction and Reproductive Genetics, \\ Shanghai, China
}

Submitted: 9 October 2017

Accepted: 11 November 2017

Arch Med Sci 2018; 14, 5: 1119-1124

DOI: https://doi.org/10.5114/aoms.2018.72790

Copyright $\odot 2018$ Termedia \& Banach

\section{Abstract}

Introduction: Preimplantation genetic diagnosis/screening (PGD/PGS) can effectively detect chromosomal abnormalities in an embryo but only if an embryo is available. However, not all couples can obtain an embryo that is available for testing. The purpose of this study was to identify factors which might affect the formation of PGD/PGS embryos to predict the possibility of obtaining embryos that could be detected.

Material and methods: In a retrospective study, we included all couples who underwent PGD/PGS at our center from January 2015 to December 2016. We compared these patients according to the non-blastocyst group and the blastocyst group.

Results: There were 302 couples who had blastocysts in their first PGD/PGS cycle. Fifty-seven couples had no blastocysts in their PGD/PGS cycles: 43 couples had no blastocysts in one cycle; 10 in two cycles; 4 in three cycles. The non-blastocyst group was older than the blastocyst group (32.37 vs. $30.69, p=0.048)$. Anti-mullerian hormone $(\mathrm{AMH}, \mathrm{ng} / \mathrm{ml})$ in the non-blastocyst group was significantly lower than in the blastocyst group (4.80 \pm 3.67 vs. $3.07 \pm 2.30, p=0.00$ ). Women whose chromosome were aneuploid (47, XXX or $45, X)$ had a similar AMH level compared with others, but the number of retrieved oocytes was much lower; the normal karyotype was 14.25 and aneuploidy was $5.40(p=0.01)$ in women $<30$ years old. There was the same condition in women aged $30-38$ years (14.60 vs. 3.44, $p<0.001$ ). Male's different chromosome karyotype had no influence on double pronuclear number or the rate of blastocyst formation. Presence of endometriosis, polycystic ovary syndrome and tubal factor showed no difference between the blastocyst and non-blastocyst group. Nor did oligospermia and asthenospermia.

Conclusions: Elderly women, those with lower AMH and women with 47, XXX or $45, X$ have fewer ova, leading to the possibility of no blastocyst. These couples should be fully informed and weigh the advantages and disadvantages before undergoing PGD/PGS.

Key words: aneuploidy, maternal age, anti-mullerian hormone, preimplantation genetic screening, preimplantation genetic diagnosis.

\section{Introduction}

Preimplantation genetic diagnosis (PGD) can solve the abortion, stillbirth and fetal malformation caused by parents' abnormal chromosome

\author{
Corresponding author: \\ Xiaoming Zhao PhD \\ Center for Reproductive \\ Medicine \\ Ren Ji Hospital \\ School of Medicine \\ Shanghai Jiao Tong University \\ No.845 Lingshan Road \\ Pudong \\ 200135 Shanghai, China \\ Phone: 86-13916308054 \\ E-mail: \\ zhao_xiao_ming@126.com
}


karyotype, while preimplantation genetic screening (PGS) provides an effective method to screen aneuploid embryos. They can reduce mental and physical pain and injury causing by curettage. However, this technology mainly adopts trophectoderm biopsy and cryopreservation of tested blastocysts for subsequent transfer, which means the blastocyst should be cultured successfully 5-7 days after fertilization. In this process, some couples' embryos have poor quality and cannot be detected. For these couples, the opportunity to detect embryos has been lost. Furthermore, ovulation and oocyte retrieval will result in physical, economic and mental costs. The purpose of this paper is to find out which factor(s) may influence the formation of blastocysts including female and male chromosome karyotype, female ovarian function and male semen. We hope to predict the possibility of having embryos tested before ovulation induction to evaluate the value of undertaking PGD/PGS.

\section{Material and methods}

\section{Subjects}

Between January 2015 and December 2016 357 couples were referred to our center seeking PGD/PGS because of male or female chromosome abnormality or recurrent miscarriage.

The abnormal karyotypes included: balanced translocation (t), Robertson translocation (rob), inversion (inv), deletion (del), mosaicism (mos) (including: 47, XX, +mar [85]/46, X, +mar [3]; 45, X [6]/46, XX [94]; 45, X [25]/46, XX [55]; 45, X [4]/46, $X X[26] ; 45, X[4] / 46, X X$ [95]), aneuploidy (47, $X X X ; 45, X)$, and ring chromosome (r). These couples attempted to undertake PGD.

Indications for PGS included advanced maternal age, repeated implantation failure and recurrent miscarriage or abnormal chorionic chromosomes being detected at least once. These couples for PGS had normal chromosome karyotype (the female was $46, X X$, and the male was $46, X Y$ ). Recurrent miscarriage was defined as 2 or more pregnancies, with early miscarriage within 12 weeks.

There was only one couple who undertook PGD for a genetic disorder, so this study excluded genetic disorders.

All couples received genetic counseling before PGD/PGS.

\section{Ovulation induction, oocyte retrieval and embryo scoring}

Ovarian stimulation was achieved using one of three protocols depending on the estimated ovarian response: agonist protocol, antagonist protocol and micro stimulation. The starting dose was determined according to various clinical factors, such as age, body mass index (BMI), FSH on day 3, anti-mullerian hormone $(\mathrm{AMH})$ and antral follicles count (AFC). Human chorionic gonadotrophin agonist triggered when at least 1 follicle was larger than $18 \mathrm{~mm}$ in diameter. Transvaginal ultrasound-guided oocyte retrieval was scheduled 34-36 $\mathrm{h}$ after hCG administration under local or general anesthesia. All oocytes obtained were inseminated by intracytoplasmic sperm injection (ICSI) to prevent residual contamination by sperm DNA. The presence of protokaryon was checked 16-18 h after fertilization. Embryos were cultured until D5-7. The blastocyst was qualified for biopsy when it was $\geq 3 A A, 3 A B, 3 B A$, $3 \mathrm{BB}$ or $\mathrm{D} 6-7$ was $\geq 4 \mathrm{AA}, 4 \mathrm{AB}, 4 \mathrm{BA}$, 4BB (Gardener scoring standard)

\section{Statistical analysis}

SPSS (version 25.0) was used for data analysis. The $\chi^{2}$ test was used for enumeration data. $T$ test and ANOVA were for quantitative data. $P<0.05$ was considered statistically significant. $P<0.01$ was considered highly statistically significant.

\section{Results}

\section{Comparison of non-blastocyst group and blastocyst group}

There were 98 couples who attempted to undertake PGS and 261 for PGD. Their chromosome karyotypes are shown in Table I.

Three hundred and two couples had blastocysts in their first PGD/PGS cycle and 57 couples had no blastocysts in their PGD/PGS cycles: 43 couples had no blastocysts in one cycles; 10 in two cycles; 4 in three cycles. Female age was significantly higher in the non-blastocyst group, $p=0.048$. There was a significant difference in $\mathrm{AMH}$ serum level $(\mathrm{ng} / \mathrm{ml})$. The non-blastocyst group had lower $\mathrm{AMH}$ and higher female age than the blastocyst group (Table II). With the times of

Table I. Chromosome karyotype in all couples

\begin{tabular}{|lccccccccccc|}
\hline Gender & Normal & $t$ & Rob & Inv & Mos & Del & Aneuploidy* & XYY & XXY & $r$ & Add \\
\hline Female & 240 & 59 & 18 & 10 & 5 & 10 & 13 & $/$ & $/$ & 1 \\
\hline Male & 189 & 85 & 26 & 21 & 2 & 9 & $/$ & 14 & 9 & 2 \\
\hline
\end{tabular}

${ }^{\star}$ Female abnormal chromosome karyotype including: 47, XX, +mar [85]/46, X, +mar [3]; 45, X [6]/46, XX [94]; 45, X [25]/46, XX [55]; 45, $X[4] / 46, X X[26] ; 45, X[4] / 46, X X[95]$. 
Table II. Comparison of non-blastocyst group and blastocyst group

\begin{tabular}{|c|c|c|c|}
\hline Parameter & Blastocyst group $(n=3020)$ & Non-blastocyst group $(n=570)$ & $P$-value \\
\hline Female age [years] & $30.69 \pm 4.1$ & $32.37 \pm 5.96$ & 0.05 \\
\hline BMI [kg/m²] & $22.42 \pm 3.35$ & $22.52 \pm 3.37$ & 0.84 \\
\hline AMH $[\mathrm{ng} / \mathrm{ml}]$ & $4.80 \pm 3.67$ & $3.07 \pm 2.30$ & $<0.001$ \\
\hline Basal FSH & $6.40 \pm 2.84$ & $6.96 \pm 3.53$ & 0.26 \\
\hline \multicolumn{4}{|l|}{ Complications (\%): } \\
\hline Endometriosis & $12(4.0)$ & $2(3.50)$ & 0.87 \\
\hline PCOS & $31(10.3)$ & $4(7.00)$ & 0.45 \\
\hline Tubal factor & $56(18.5)$ & $12(21.10)$ & 0.66 \\
\hline \multicolumn{4}{|l|}{ Semen condition (\%): } \\
\hline Normal & $163(53.97)$ & $35(61.40)$ & 0.30 \\
\hline $\begin{array}{l}\text { Oligospermia and/or } \\
\text { asthenospermia }\end{array}$ & $139(46.03)$ & $22(38.60)$ & \\
\hline
\end{tabular}

failure of obtaining a blastocyst, the $\mathrm{AMH}$ level gradually decreased (Figure 1). Average AMH was 5.34 in the blastocyst group. Average AMH was 3.41 for no blastocyst for once, 2.73 for twice and 2.12 for three times.

Presence of endometriosis, PCOS and tubal factor showed no difference between the blastocyst and non-blastocyst group.

Nor did the proportion of oligospermia and asthenospermia.

\section{Influence of different female chromosome karyotype on embryo formation}

We re-divided three groups according to female age in order to correct the age deviation and found that not only in women who were under 30 years but also in those 30-38 years old, the $\mathrm{AMH}$ value was similar in women with different chromosome karyotypes. However, the number of retrieved oocytes was significantly lower in aneuploid females.

For females less than 30 years old, aneuploid females (8) received $5.40 \pm 3.65$ oocytes, while normal women (34) obtained $14.25 \pm 5.23$, and balanced translocation women (8) had 12.68 \pm 7.82 . Robertson translocation women (11) had $16.30 \pm 8.35$ oocytes, inversion (7) women 10.43 \pm 5.59 oocytes, women with deletion (4) had 14.25 \pm 3.06 oocytes, and 1 woman with ring chromosome obtained 18 oocytes (Figure 2).

For females 30 to 38 years old, the number of retrieved oocytes was $3.44 \pm 3.05$ in aneuploid females (9). In normal chromosome women (61) the number was $12.30 \pm 7.30$, in balanced translocation women (37) it was $10.73 \pm 5.53$, in Robertson translocation women (9) it was $6.33 \pm 3.16$, in inversion women (3) it was $15.00 \pm 3.64$, in deletion

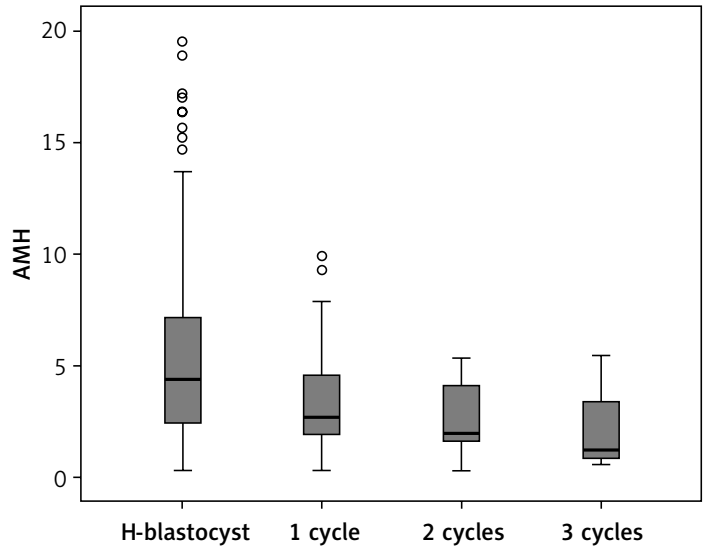

Figure 1. Relationship between times of failure of having blastocyst and $\mathrm{AMH}$ value

$\mathrm{H}$-blastocyst means having a blastocyst, 1 cycle means 1 cycle of not getting a blastocyst, 2 cycles means 2 cycles of not getting a blastocyst, 3 cycles means 3 cycles of not getting a blastocyst. The greater the number of times of failing to get a blastocyst, the lower was the AMH value.

women (10) it was $9.33 \pm 6.24$, and in mosaicism women (4) it was $14.75 \pm 9.57$ (Figure 2).

For females over 38 years old, normal women obtained $6.67 \pm 4.66$ oocytes, balanced translocation women (5) had $4.80 \pm 1.64$, and 1 woman with inversion obtained 8 oocytes (Figure 2).

There were no significant differences in MII rate, $2 \mathrm{PN}$ rate, or blastocyst formation rate between the three groups.

\section{Influence of different male chromosome karyotype on embryo formation}

The $\mathrm{AMH}$ value, the number of oocytes, the number of normal fertilizations and the number of blastocysts formed were not significantly different among men with different karyotypes (Table III). 
Table III. ANOVA was used to analyze AMH, the number of oocytes, the number of normal fertilized eggs and the number of blastocysts formed in different male chromosomes of the females of different age groups

\begin{tabular}{|lccc|}
\hline Parameter & \multicolumn{3}{c|}{ Females' age } \\
\cline { 2 - 4 } & $<30$ & $30-38$ & $>38$ \\
\hline AMH & 0.01 & 0.37 & 0.80 \\
\hline No. of oocytes & 0.12 & 0.68 & 0.59 \\
\hline Normal fertilized egg & 0.69 & 0.90 & 0.72 \\
\hline No. of blastocysts & 0.87 & 0.86 & 0.55 \\
\hline
\end{tabular}

A

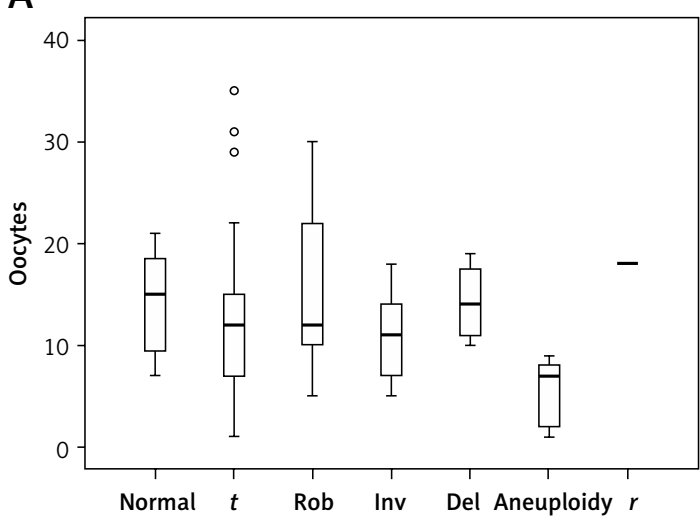

C

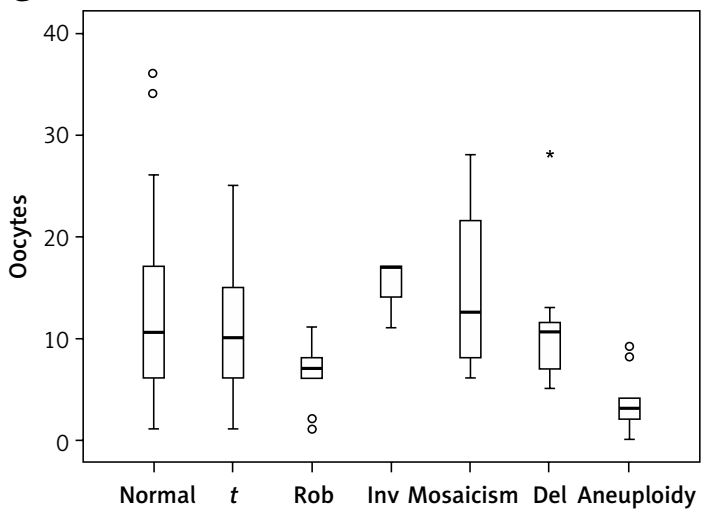

E

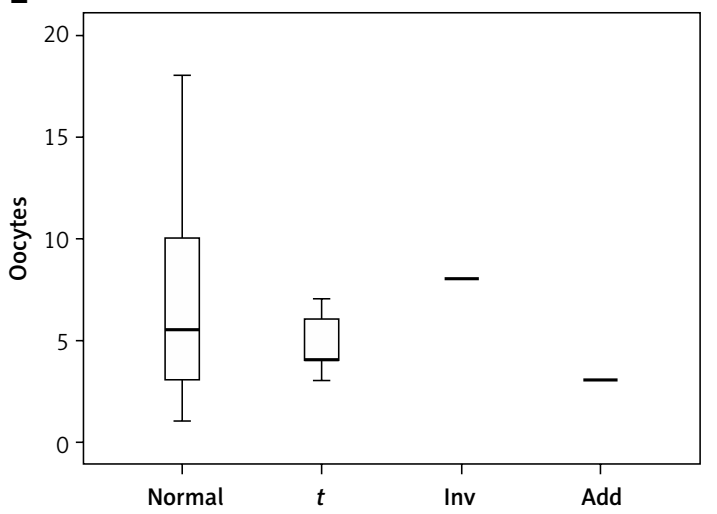

\section{Discussion}

\section{$\mathrm{AMH}$ and blastocyst formation}

As women's age increases, their fertility decreases gradually due to exogenous or endogenous multifactorial effects, such as life style, and/ or endocrine disrupting chemicals. Women showing a limited number of oocytes following ovum pick-up, especially MII oocytes, present impaired embryo production and, therefore, decreased chances of pregnancy [1]. It was reported that the IVF cancellation rate was $22 \%$ in women older than $40.17 .6 \%$ of these women had a biochem-

B

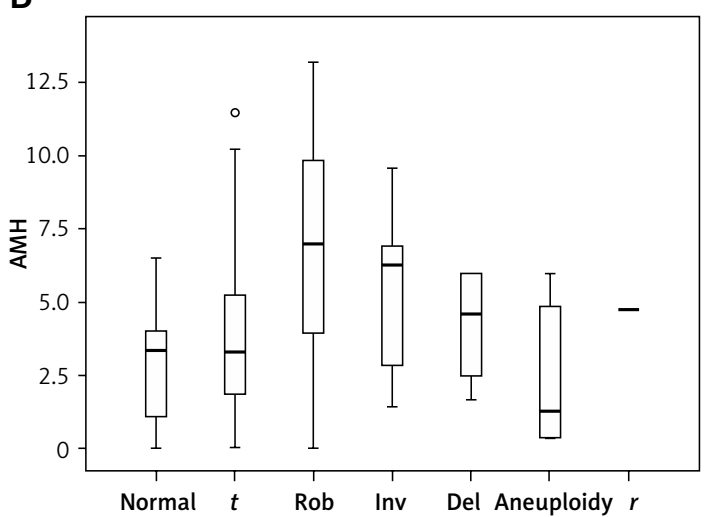

D

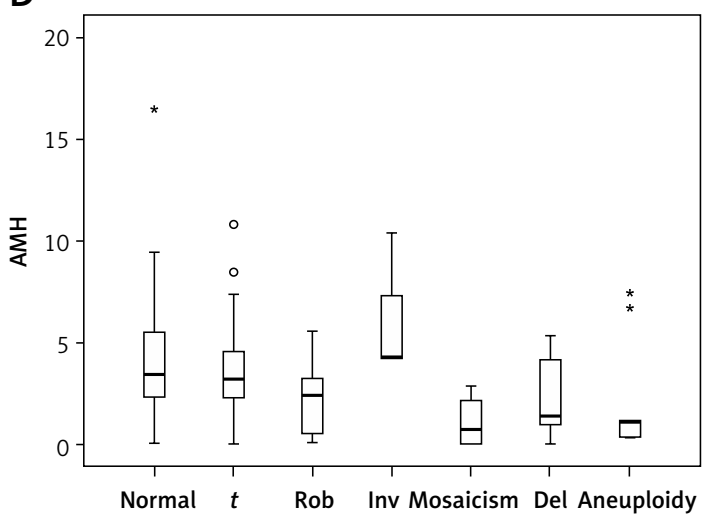

$\mathrm{F}$

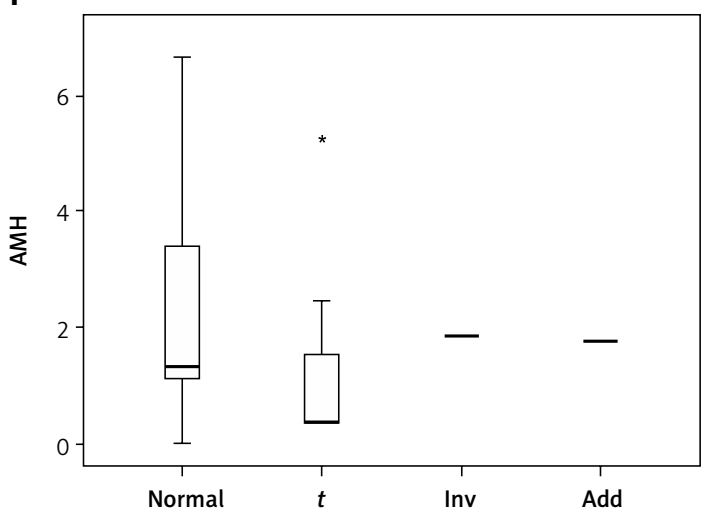

Figure 2. Comparison of $A M H$ and ovum number between different chromosomes of the female $(A M H: n g / m l):$ A, B - female $<30$ years old, $\mathbf{C}, \mathbf{D}-$ female $\geq 30$ and $\leq 38$ years old, $\mathbf{E}, \mathbf{F}-$ female $>38$ years old 
ical pregnancy in IVF and $49 \%$ of these women had early miscarriage [2]. It was also reported that in women $>40$ the live birth rate was $4.7 \%$ and the pregnancy rate was even lower (1.6-0\%) when more than 44 years old [3-6]. Therefore, the woman's age is critical for the formation of PGS embryos. This may be related to antral follicle count and mitochondria in the female granulosa cells. In elderly women, ovarian function and the number of oocytes decreased. In addition, the rate of aneuploidy is lower than that in normal ovarian reserve $[4,7,8]$. The higher the mitochondrial DNA content in female granulosa cells, the better is the embryo quality. However, as women age, mitochondrial loss increases, and embryo quality decreases gradually [9]. Thus, for older women, both cleavage phase embryos and the D5 or D6 blastocysts which can be diagnosed by genetics will significantly decrease, as age increases [10, 11].

Anti-mullerian hormone is one of the best endocrine markers for assessing age-related fertility, which is more sensitive than basal FSH. Anti-mullerian hormone is positively related to the number of antral follicles: the more antral follicles there are, the higher is the serum concentration of $\mathrm{AMH}$, which is also related to the number of obtained oocytes, MII oocytes and embryos [9]. In our study, it was found that $\mathrm{AMH}$ was one of the important factors determining whether the embryos could be tested. The AMH in the non-blastocyst group is lower than in the blastocyst group. Women with low $\mathrm{AMH}$ may not be able to have a blastocyst to be detected even if they try two or three ovulation induction cycles. At the same time, even in women with normal ovarian function, the level of $\mathrm{AMH}$ has an impact on the number of embryos that can be detected.

\section{Female abnormal karyotype and blastocyst formation}

In a PGD study of women with 33 pairs of autosomal balanced translocations, it was found that the number of retrieved oocytes, D3 embryos and the COS parameters were not significantly different from those of normal female subjects, and the pregnancy rates of the two groups were similar. It showed that female chromosome balance translocation had no effect on ovarian response [12]. The study also found only two factors affecting ovarian function: AMH and the sex of the balanced translocation. Keymolen et al. also reported women's ovarian function in balanced translocation was not diminished [13]. They reported that 76 couples underwent 124 cycles with similar numbers of eggs, with similar rates of pregnancy per transplant/oocyte cycle, and concluded that sex had no effect on ovarian response and PGD outcome. The ESHRE consensus also referred to the 3524 PGD oocyte cycle; because of chromosomal abnormalities, the sex of the balanced translocation had no effect on the pregnancy rate [14].

However, it has been reported that some autosomal Robertson translocations and inversions can lead to ovarian dysfunction, especially if the balanced translocation involves sexual chromosomes [15]. In our study, we found that only when the number of chromosome $X$ is abnormal (47, XXX and $45, X)$, these women's oocytes number was the lowest, while the MII rate and normal fertilization rate were not significantly different, but the low number of oocytes will cause lower blastocyst formation, even to zero. Other female chromosomal abnormalities such as balanced translocation, Robertson translocation, and inversion did not show any difference in the number of retrieved eggs, the number of MIl eggs, the number of normal fertilizations, or the number of blastocysts formed. The presence of karyotypic X chromosome abnormalities can affect the ovarian function, e.g. the female $X$ chromosome and autosome translocation will appear [16]. Turner syndrome in women with premature ovarian failure $(45, X)$ will show congenital ovarian hypoplasia or premature ovarian failure. $X$ chromosome fragile ovarian function in women also fell [17]. But once these women acquire embryos, their pregnancy rates are similar.

Our results supported the above findings and showed that is not true for mosaic of $X$. The mosaic of $X$ in this study included $47, X X,+\operatorname{mar}[85] / 46$, $X,+\operatorname{mar}[3], 45, X[6] / 46, X X[94], 45, X[25] / 46$, $X X$ [55], 45, X [4]/46, XX [95], XX [26]. Even if the highest percentage of mosaic was $31.5 \%$ (45, $X$ [25]/46, XX [55]), it did not affect the number of oocytes obtained, and also detectable blastocysts could be obtained.

\section{Male abnormal karyotype and blastocyst formation}

Huang and other researchers have found that oligospermia and asthenospermia are the major causes of male Robertson translocation. Of the 80 men who had Robertson translocation, 70 were infertile due to men's oligospermia, accounting for $87.55 \%$ [18]. The chromosomes of the human Robertson translocation carriers are 13, 14, 15, 21 and 22 , and do not involve the $Y$ chromosome, which is closely related to spermatogenesis. Eaker established a mouse model of spermatogenic cells carrying Robertson translocation, and observed that the Robertsonian translocation carrying mouse spermatogenic cell apoptosis ratio increased, and centromere protein expression was significantly increased, suggesting that the chromosomes of spermatogenic cells in mice have abnormal Robertson translocation in the arrangement of cell division [19]. It may be due to abnormal meiosis 
that leads to cell arrest or spermatogenesis damage [20], so the man has an autosomal balanced translocation and also appears to have severe oligozoospermia [21].

In our study, however, there were no statistically significant differences in the proportion of men with or without spermatozoa between the blastocyst group and those without blastocysts, and a normal proportion of semen in the males. There were no significant differences in normal fertilization rates and blastocyst formation rates between males with different chromosomal abnormalities. Even if the number of sex chromosomes involved was abnormal, including 47, XXY, and 47, XYY, the normal number of fertilizations and the number of blastocysts formed were not significantly different from those of normal men. Although the male chromosome abnormality may have an adverse effect on spermatogenesis, it is speculated that PGD using intracytoplasmic sperm injection could reduce the adverse effects on fertilization of small and weak sperm and had no significant effect on embryo formation.

In conclusion, we conclude that women's age and ovarian function are determining factors to obtain a detectable blastocyst. In addition, when the chromosomal abnormalities involves the $X$ chromosome (47, XXX or $45 \mathrm{X})$, it will also make the number of oocytes decreased. So, if the woman is older, with poor ovarian function and/or the chromosome aneuploid (47 XXX or $45 \mathrm{X}$ ), she should be fully informed of the fewer number of oocytes and the high possibility of blastocyst culture failure before undertaking PGD/PGS. Medical staff and patients should consider a more suitable method for assisted reproduction.

\section{Acknowledgments}

This project was supported by the National Science Foundation of China (Grant No. 81370687).

The authors wish to thank all the staff in the reproductive medicine center who made an effort to complete all the PGD/PGS cycles.

\section{Conflict of interest}

The authors declare no conflict of interest.

\section{References}

1. Nich M, de Cassia Savio Frigueira R, de Almeida Ferreira Braga D, Souza Setti A, laconelli A Jr, Borges E Jr. Decreased fertility in poor responder women is not related to oocyte morphological status. Arch Med Sci 2011; 7 : 315-20.

2. Cabry R, Merviel P, Hazout A, et al. Management of infertility in women over 40. Maturitas 2014; 78: 17-21.

3. Tsafir A, Simon A, Revel A, Reubinoff B, Lewin A, Laufer N Retrospective analysis of 1217 IVF cycles in women aged 40 aged old and older. RBM online 2007; 14: 348-55.
4. Klipstein S, Regan M, Ryley D, Goldman M, Alper M, Reindollar R. One last chance for pregnancy: a review of 2705 in vitro fertilization cycles initiated in women age 40 years and above. Fertil Steril 2005; 84: 435-45.

5. Ron-El R, Raziel A, Strassburger D, Schachter M, Kasterstein E, Friedler S. Outcome of assisted reproductive technology in women over the age of 41 . Fertil Steril 2000; 74: 471-5.

6. Ciray HN, Ulug U, Tosun S, Erden HF, Bahceci M. Outcome of 1114 ICSI and embryo transfer cycles of women 40 years of age and over. Reprod Biomed Online 2006; 13: 516-22.

7. Suchartwatnachai C, Wongkularb A, Srisombut C, Choktanasiri W, Chinsomboon S, Rojanasakul A. Cost-effectiveness of IVF in women 38 years and older. Int J Gynecol Obstet 2000; 69: 143-8.

8. Toner J. Age=egg quality, FSH level=egg quantity. Fertil Steril 2003; 79: 491.

9. Scheffer JB, Scheffer BB, de Carvalho RF, et al. Age as a predictor of embryo quality regardless of the quantitative ovarian response. Int J Fertil Steril 2017; 11: 40-6.

10. McCoy RC, Demkok ZP, Ryan A, et al. Evidence of selection against complex mitotic-origin aneuploidy during preimplantation development. PLoS Genetics 2015; 22: 11.

11. Desquiret-Dumas V, Clement A, Seegers V, et al. The mitochondrial DNA content of cumulus granulosa cells is linked to embryo quality. Hum Reprod 2017; 32: 607-14.

12. Dechanet C, Castelli D, Reyftmann L, et al. Do female translocations influence the ovarian response pattern to controlled ovarian stimulation in preimplantation genetic diagnosis? Hum Reprod 2011; 25: 1232-40.

13. Keymolen K, Staessen C, Verpoest W, et al. A proposal for reproductive counselling in carriers of Roberstonian translocations: 10 years of experience with preimplantation genetic diagnosis. Hum Reprod 2009; 24: 2365-71.

14. Goossens V, Harton G, Moutou C, Traeger-Synodinos J, Van Rij M, Harper JC. ESHRE PGD Consortium data collection IX: cycles from January to December 2006 with pregnancy follow-up to October 2007. Human Reprod 2009; 24: 1786-810.

15. Kummer N, Marin JR, Pal L. Diminished ovarian reserve in a woman with a balanced 13;21 translocation. Fertil Steril 2009; 24: 2365-71.

16. Schlessinger D, Herrela L, Crisponi L, et al. Genes and translocations involved in POF. Am J Med Genet 2002; 111: 328-33.

17. Tsafrir A, Sltarescu G, Margolioth E, et al. PGD for fragile $X$ syndrome: ovarian function is the main determinant of success. Hum. Reprod 2010; 25: 2629-36.

18. Huang J, Lian Y, Qiao J, Liu P. Clinical characteristics and preimplantation genetic diagnosis for male Robertsonian translocations. J Peking Univ (Health Sciences) 2012; 44: 544-6.

19. Eaker S, Pyle A, Cobb J, Handel MA. Evidence for meiotic spindle checkpoint from analysis of spermatocytes from Robertsonian-chromosome heterozygous mice. J Cell Sci 2001; 114: 2953-65.

20. Leng $M$, Li G, Zhong L, et al. Abnormal synapses and recombination in an azoospermic male carrier of a reciprocal translocation $\mathrm{t}(1 ; 21)$. Feril Steril 2009; 91: 17-22.

21. Rao KL, Babu KA, Kanakavalli MK, Padmalatha VV, Deenadayal M, Singh L. Prevalence of chromosome defects in azoospermic and oligoastheno-teratozoospermic South Indian infertile men attending an infertility clinic. Reprod Biomed Online 2005; 10: 467-72. 